\title{
Light Cluster Production in Central Symmetric Heavy-Ion Reactions from Fermi to Gev Energies
}

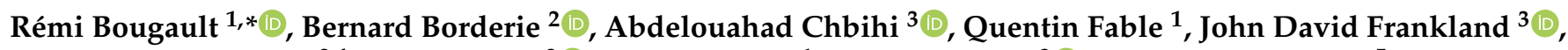 \\ Emmanuelle Galichet ${ }^{2,4}$, Tom Genard ${ }^{3}{ }^{1}$, Diégo Gruyer ${ }^{1}$, Maxime Henri ${ }^{3}{ }^{\circledR}$, Marco La Commara ${ }^{5}$,

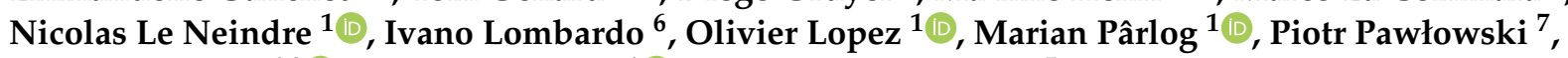 \\ Giuseppe Verde ${ }^{6,8}{ }^{(\mathbb{D}}$, Emmanuel Vient $^{1}{ }^{1}$ and Mariano Vigilante ${ }^{5}$
}

1 Normandie University, ENSICAEN, UNICAEN, CNRS/IN2P3, LPC, 14050 Caen, France; fable@lpccaen.in2p3.fr (Q.F.); gruyer@lpccaen.in2p3.fr (D.G.); leneindre@lpccaen.in2p3.fr (N.L.N.); lopezo@lpccaen.in2p3.fr (O.L.); parlog@lpccaen.in2p3.fr (M.P.); vient@lpccaen.in2p3.fr (E.V.)

2 Université Paris-Saclay, CNRS/IN2P3, IJCLab, 91405 Orsay, France; bernard.borderie@ijclab.in2p3.fr (B.B.); galichet@ipno.in2p3.fr (E.G.)

3 Grand Accélérateur National d'Ions Lourds (GANIL), CEA/DRF-CNRS/IN2P3, 14076 Caen, France; abdelouahad.chbihi@ganil.fr (A.C.); john.frankland@ganil.fr (J.D.F.); tom.genard@ganil.fr (T.G.); maxime.henri@ganil.fr (M.H.)

4 Conservatoire National des Arts et Metiers, 75141 Paris, France

5 Dipartimento di Fisica 'E. Pancini' and Sezione INFN, Universitá di Napoli 'Federico II', 80126 Naples, Italy; marco.lacommara@na.infn.it (M.L.C.); vigilant@na.infn.it (M.V.)

6 Istituto Nazionale di Fisica Nucleare (INFN), Sezione di Catania, 95123 Catania, Italy; ivano.lombardo@ct.infn.it (I.L.); giuseppe.verde@ct.infn.it (G.V.)

check for updates

Citation: Bougault, R.; Borderie, B.; Chbihi, A.; Fable, Q.; Frankland, J.D.; Galichet, E.; Genard, T.; Gruyer, D.;

Henri, M.; La Commara, M.; et al. Light Cluster Production in Central Symmetric Heavy-Ion Reactions from Fermi to Gev Energies. Symmetry 2021, 13, 1406. https://doi.org/ $10.3390 /$ sym 13081406

Academic Editor: Andrea Lavagno

Received: 29 June 2021

Accepted: 20 July 2021

Published: 2 August 2021

Publisher's Note: MDPI stays neutral with regard to jurisdictional claims in published maps and institutional affiliations.

Copyright: (c) 2021 by the authors. Licensee MDPI, Basel, Switzerland. This article is an open access article distributed under the terms and conditions of the Creative Commons Attribution (CC BY) license (https:// creativecommons.org/licenses/by/ $4.0 /)$.
7 Institute of Nuclear Physics, Polish Academy of Sciences, 31-342 Krakow, Poland; piotr.pawlowski@ifj.edu.pl

8 Laboratoire des 2 Infinis Toulouse, Université de Toulouse, CNRS/IN2P3, UPS, 31000 Toulouse, France

* Correspondence: bougault@lpccaen.in2p3.fr

Abstract: Correlations and clustering are of great importance in the study of the Nuclear Equation of State. Information on these items/aspects can be obtained using heavy-ion reactions which are described by dynamical theories. We propose a dataset that will be useful for improving the description of light cluster production in transport model approaches. The dataset combines published and new data and is presented in a form that allows direct comparison of the experiment with theoretical predictions. The dataset is ranging in bombarding energy from 32 to $1930 \mathrm{~A} \mathrm{MeV}$. In constructing this dataset, we put in evidence the existence of a change in the light cluster production mechanism that corresponds to a peak in deuteron production.

Keywords: nuclear physics; heavy-ion reactions; Nuclear Equation of State; light cluster production; transport models

\section{Introduction}

Knowledge of the mechanism of fragment and light cluster formation in heavy-ion collisions allows us to trace the fundamental properties of nuclear matter [1]. In nuclear collisions, the emission of several fragments from a hot nucleus, the multifragmentation process, has been observed, and the fragment characteristics are well described by statistical concepts. However, in multifragmentation models, the description of the total observed production rates of light particles does not reach, by far, the accuracy obtained for the heavier fragments. In heavy-ion transport models, two main actors are at work: the onebody approach (mean field) and the few-body correlation in a medium (clustering). The formation of clusters and fragments is determined by the proper treatment of correlations and the proper introduction and propagation of fluctuations in dynamical models. It turns out that a problem exists concerning light cluster description in many transport approaches. This problem has been addressed, for example, in [2], with the dynamic production of $\mathrm{A} \leq 3$ fragments and in [1] by considering wave-packet splitting and by taking into account 
the cluster correlations explicitly. In all cases, the calculations have shown that taking into account the clustering is important since many observables depend on it.

The aim of the present article is to provide experimental data on cluster production to which the model calculations can be directly compared in order to improve our understanding of the Nuclear Equation of State. It is also useful to recall that light nuclear clusters play an important role in the warm and low-density nuclear matter that can be found in core-collapse supernovae and neutron star mergers [3], and therefore, their production mechanism is also important for astrophysics.

\section{Materials and Methods}

The $4 \pi$ multi-detector INDRA [4] was used to study four nuclear reactions with beams of ${ }^{58} \mathrm{Ni}$ accelerated by the GANIL cyclotrons (Caen) to 32, 52, 64 and $74 \mathrm{MeV} /$ nucleon and a thin $\left(179 \mu \mathrm{g} / \mathrm{cm}^{2}\right)$ target of ${ }^{\text {nat }} \mathrm{Ni}$ and two reactions with beams of ${ }^{197} \mathrm{Au}$ accelerated by the heavy-ion synchrotron SIS at GSI (Darmstadt) to 40 and $60 \mathrm{MeV} /$ nucleon and a $2000 \mu \mathrm{g} / \mathrm{cm}^{2}$ target of ${ }^{n a t} \mathrm{Au}$. Higher beam energy experiments were performed during these experimental campaigns, but they were excluded from the present analysis because of the limited stopping power of the experimental INDRA apparatus to high energetic light-charged particles produced in the forward direction.

INDRA is a charged product multidetector composed of 336 detection cells arranged in 17 rings centered on the beam axis and covering $90 \%$ of the solid angle. The first ring $\left(2-3^{\circ}\right)$, made of 12 phoswich telescopes, was not used in the $\mathrm{Ni}+\mathrm{Ni}$ analysis due to a malfunction during the experiment. For the $\mathrm{Au}+\mathrm{Au}$ experimental campaign, the phoswich telescopes $\left(2-3^{\circ}\right)$ were replaced by 12 telescopes each one composed of $300 \mu \mathrm{m}$ silicon wafer $(\mathrm{Si})$ and a $\mathrm{CsI}(\mathrm{Tl})$ scintillator $\left(14 \mathrm{~cm}\right.$ thick). Rings $2-9\left(3-45^{\circ}\right)$ are composed of 12 or 24 three-member detection telescopes; a $5 \mathrm{~cm}$ thick ionization chamber (50 mbar); a $300 \mu \mathrm{m}$ silicon wafer; and a CsI(Tl) scintillator (14-10 cm thick) coupled to a photomultiplier tube. Rings 10-17 $\left(45^{\circ}\right.$ to $\left.176^{\circ}\right)$ are composed of 24,16 or 8 two-member telescopes; an ionization chamber ( $5 \mathrm{~cm}$ thick, $30 \mathrm{mbar}$ ); and a CsI(Tl) scintillator of 8,6 or $5 \mathrm{~cm}$ thickness. INDRA can identify in charge fragments from Hydrogen to Uranium and in mass light fragments $(Z \leq 4)$ with low thresholds. The recorded event functionality was activated under a triggering factor based on a minimum number of fired telescopes $\left(\mathrm{N}^{\min }\right)$ over the detector acceptance $(90 \%$ of $4 \pi)$. During the $\mathrm{Ni}+\mathrm{Ni}$ experiments, $\mathrm{N}^{\min }$ was set to 4 , while, during the $\mathrm{Au}+\mathrm{Au}$ experiments, $\mathrm{N}^{\text {min }}$ was 3 .

The goal of the present article is to extend to low bombarding energies some of the results presented in an article published by the FOPI collaboration [5]: the yields of light clusters contained in Appendix. The FOPI article "Systematics of Central Heavy Ion Collisions in the $1 \mathrm{~A} \mathrm{GeV}$ Regime" presents data concerning 25 system-energies from $90 \mathrm{~A}$ $\mathrm{MeV}$ to $1.93 \mathrm{~A} \mathrm{GeV}$ bombarding energies. Because for a given bombarding energy cluster, yields depend on projectile and target isotopic composition [6], we will use the systems listed in Table 1 to perform the comparison.

Table 1. A list of the systems (projectile + target) retained for the present analysis. The data set refers to the used experimental apparatus. The last row presents the neutron-to-proton ratio of the total combined system.

\begin{tabular}{ccccc}
\hline Projectile and Target & ${ }^{58} \mathbf{N i}+{ }^{n a t} \mathbf{N i}$ & ${ }^{58} \mathbf{N i}+{ }^{58} \mathbf{N i}$ & ${ }^{40} \mathrm{Ca}+{ }^{40} \mathrm{Ca}$ & ${ }^{197} \mathrm{Au}+{ }^{n a t} \mathbf{A u}$ \\
\hline Data set & INDRA & FOPI & FOPI & INDRA and FOPI \\
$\mathrm{N} / \mathrm{Z}$ & 1.084 & 1.071 & 1.000 & 1.493 \\
\hline
\end{tabular}

The small $\mathrm{N} / \mathrm{Z}$ difference between $\mathrm{Ni}+\mathrm{Ni}$ and $\mathrm{Ca}+\mathrm{Ca}$ indicates that these two data sets can be aggregated when using independent system size variables. 


\section{Results}

\subsection{Central Event Selection and Cluster Mean Multiplicities}

The FOPI data (yield tables presented in Appendix of [5]) correspond to a centrality selection with an estimated upper limit of the reduced impact parameter $\left(b_{0}\right)$ of 0.15 . Furthermore, careful work of interpolations and extrapolations has been performed so as to present the event topology in full $4 \pi$ coverage from detected events. This last point explains the rather large FOPI multiplicity uncertainties presented in Appendix of Reference [5].

For INDRA data, the total transverse energy of detected light-charged particles (lcp, $\mathrm{Z}=1$ and 2$),\left(\Sigma E_{t}\right)^{l c p}$, is chosen as an impact parameter selector, and, as for the FOPI data, centrality selections were defined using the sharp cut-off approximation of [7] in order to have estimated reduced impact parameters for central events $b_{0}<0.15$. For INDRA data, the lcp yields are calculated using only the forward part of the center of mass (hereinafter called c.m.). The multi-detector, for these reactions, possesses better detection performances in the forward c.m. half hemisphere as compared to the backward part. Therefore, if necessary, the total detected yields can be estimated by doubling the values.

In Tables 2 and 3 , for each system, the $\left(\Sigma E_{t}\right)^{l c p}$ threshold value used for central event selection and the mean value of cluster multiplicities detected in the forward c.m. part are presented. The associated uncertainty values correspond to the error on the mean value determination (standard error on the mean).

Table 2. Ni + Ni central events (INDRA) for four bombarding energies: threshold values of the total transverse energy of detected light-charged particles that correspond to a reduced impact parameter of 0.15 . Mean values of cluster multiplicities detected in the forward c.m. part and their associated uncertainties.

\begin{tabular}{|c|c|c|c|c|}
\hline${ }^{58} \mathrm{Ni}+{ }^{n a t} \mathrm{Ni}\left(\mathrm{b}_{0}<0.15\right)$ & $32 \mathrm{~A} \mathrm{MeV}$ & $52 \mathrm{~A} \mathrm{MeV}$ & $64 \mathrm{~A} \mathrm{MeV}$ & $74 \mathrm{~A} \mathrm{MeV}$ \\
\hline$\overline{\left(\Sigma E_{t}\right)^{l c p}>}$ & $225 \mathrm{MeV}$ & $350 \mathrm{MeV}$ & $425 \mathrm{MeV}$ & $500 \mathrm{MeV}$ \\
\hline$\left(M_{1_{H}}\right)_{\text {forwar }}$ & $3.000 \pm 0.003$ & $3.582 \pm 0.003$ & $3.866 \pm 0.003$ & $3.972 \pm 0.003$ \\
\hline$\left(M_{2}\right)_{\text {forwar }}$ & $1.049 \pm 0.002$ & $1.583 \pm 0.002$ & $1.823 \pm 0.002$ & $1.948 \pm 0.002$ \\
\hline$\left(M_{3}\right)_{\text {forward c.m. }}$. & $0.447 \pm 0.001$ & $0.753 \pm 0.002$ & $0.958 \pm 0.002$ & $1.090 \pm 0.002$ \\
\hline$\left(M_{3} \mathrm{He}\right)_{\text {forward c.m. }}$. & $0.340 \pm 0.001$ & $0.570 \pm 0.001$ & $0.695 \pm 0.001$ & $0.793 \pm 0.002$ \\
\hline$\left(M_{4}{ }^{2}\right)$ forward c.m. & $3.116 \pm 0.003$ & $3.491 \pm 0.004$ & $3.604 \pm 0.003$ & $3.557 \pm 0.003$ \\
\hline
\end{tabular}

Table 3. Au + Au central events (INDRA) for two bombarding energies: threshold values of the total transverse energy of detected light-charged particles which correspond to a reduced impact parameter of 0.15 . Mean values of cluster multiplicities detected in the forward c.m. part and their associated uncertanties.

\begin{tabular}{ccc}
\hline${ }^{197} \mathrm{Au}+{ }^{\text {nat }} \mathbf{A u}\left(\mathbf{b}_{\mathbf{0}}<\mathbf{0 . 1 5}\right)$ & $\mathbf{4 0} \mathbf{A ~ M e V}$ & $\mathbf{6 0} \mathbf{~ A ~ M e V}$ \\
\hline$\left(\Sigma E_{t}\right)^{l c p}>$ & $625 \mathrm{MeV}$ & $1050 \mathrm{MeV}$ \\
$\left(M_{1_{H}}\right)_{\text {forward c.m. }}$ & $4.016 \pm 0.005$ & $5.185 \pm 0.003$ \\
$\left(M_{2}\right)_{\text {forward c.m. }}$ & $2.484 \pm 0.004$ & $3.412 \pm 0.002$ \\
$\left(M_{3}\right)_{\text {forward }}$.m. & $2.107 \pm 0.004$ & $2.837 \pm 0.002$ \\
$\left(M_{3}\right)_{\text {forward c.m. }}$ & $0.731 \pm 0.002$ & $1.179 \pm 0.002$ \\
$\left(M_{4} H e\right)$ forward c.m. & $5.843 \pm 0.002$ & $6.816 \pm 0.003$ \\
\hline
\end{tabular}

\subsection{Cluster Production: Abundance Ratios}

As mentioned previously, the FOPI data is extrapolated to full $4 \pi$ coverage, and therefore, it is impossible to directly compare the mean multiplicity values presented in [5] to INDRA data presented in Tables 2 and 3. The FOPI data is extrapolated because the detection efficiency varies according to the bombarding energy. If this variation is not corrected, the different bombarding energy data can hardly be compared. This variation according to bombarding energy is also true for INDRA data. An example of this defect can 
be seen by comparing the INDRA multiplicity of ${ }^{4} \mathrm{He}$ for the 64 and $74 \mathrm{~A} \mathrm{MeV} \mathrm{Ni}+\mathrm{Ni}$ systems (Table 2): the multiplicity is decreasing as the bombarding energy is increasing simply because of the absence of a detector below three degrees in the laboratory reference frame.

We have chosen not to extrapolate the INDRA data using the cluster abundance ratios [8]. One way around the varying detection efficiency is to compare the cluster mean multiplicities relative to the proton mean multiplicity (hereafter called cluster abundance ratios). By doing so for each system, we expect that the detection efficiency variation is largely canceled out since it is present in both the numerator and the denominator of the cluster abundance ratio. The polar angular area not covered by INDRA is from 0 to 3 degrees for $\mathrm{Ni}+\mathrm{Ni}$ and 0 to 2 degrees for $\mathrm{Au}+\mathrm{Au}$. This is a source of systematic errors in addition to the statistical errors presented in Tables 2 and 3 to calculate the total abundance ratios uncertainties if those ratios were calculated with extrapolated $4 \pi$ multiplicities. In the present case, we do not take this into account because the following figures show the values of the INDRA ratios calculated with the measured multiplicities. The use of abundance ratios, or even other ratios, allows us also to compare the FOPI full $4 \pi$ coverage data set to the INDRA forward c.m. detected data set. The other advantage lies in the fact that ratios remove also trivial size dependency [5], and therefore, we will be authorized to aggregate $\mathrm{Ni}+\mathrm{Ni}$ with $\mathrm{Ca}+\mathrm{Ca}$ results since these systems have almost the same global neutron-to-proton ratio (which we will hereafter call isospin).

${ }^{2} \mathrm{H},{ }^{3} \mathrm{H},{ }^{3} \mathrm{He}$ and ${ }^{4} \mathrm{He}$ abundance ratios are presented in Figure 1 for $\mathrm{Ni}+\mathrm{Ni}$ and $\mathrm{Ca}+\mathrm{Ca}$ systems and in Figure 2 for $\mathrm{Au}+\mathrm{Au}$.

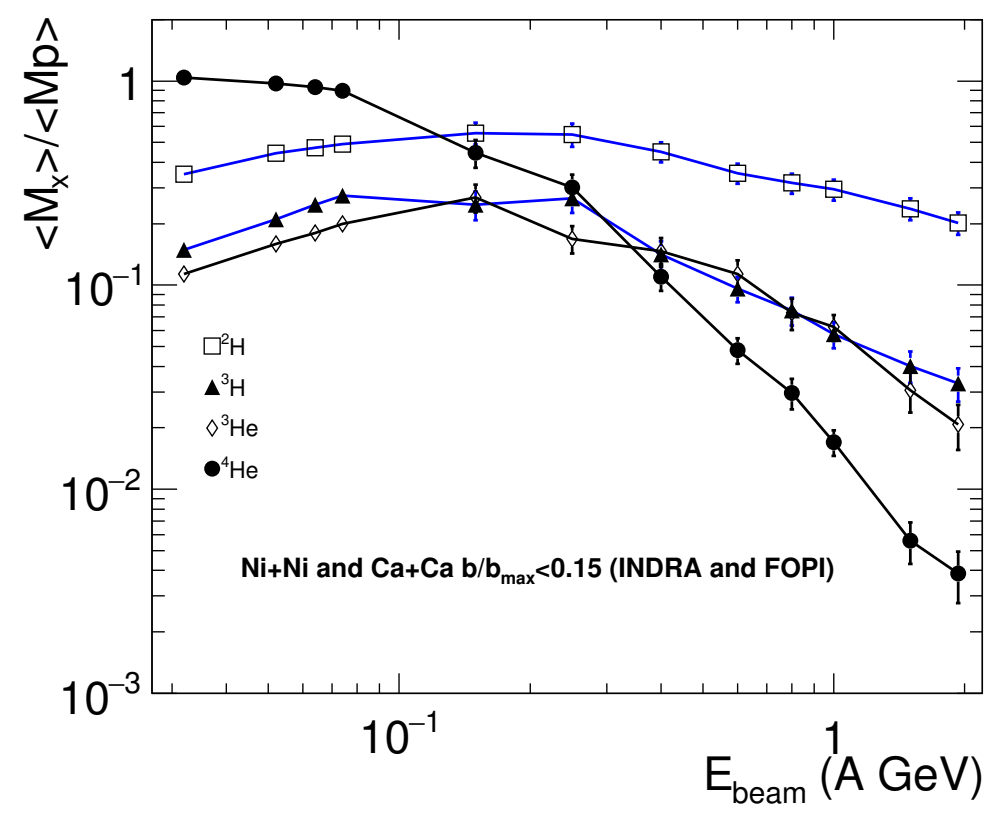

Figure 1. Light cluster abundance ratios as a function of bombarding energy for $\mathrm{Ni}+\mathrm{Ni}$ and $\mathrm{Ca}+\mathrm{Ca}$ systems. Lines to guide the eye. Line colours are to differentiate them.

In Figure 1, the bombarding energy data points are ranging from 32 to $1930 \mathrm{~A} \mathrm{MeV}$. The FOPI Ca + Ca data starts at $400 \mathrm{~A} \mathrm{MeV}$. Below $400 \mathrm{~A} \mathrm{MeV}$, the data are $\mathrm{Ni}+\mathrm{Ni}$ systems, and the first four values concern INDRA data set. The absence of discontinuity between values concerning $\mathrm{Ni}+\mathrm{Ni}$ and $\mathrm{Ca}+\mathrm{Ca}$ systems confirms the fact that the use of ratios eliminates trivial size effects.

In Figure 2, the bombarding energy data points are ranging from 40 to $1500 \mathrm{~A} \mathrm{MeV}$. The FOPI data starts at $90 \mathrm{~A} \mathrm{MeV}$, for which ${ }^{3} \mathrm{He}$ and ${ }^{4} \mathrm{He}$ multiplicity values are not available in the published article. HADES data for ${ }^{2} \mathrm{H},{ }^{3} \mathrm{H}$ and ${ }^{3} \mathrm{He}[9]$ at $1230 \mathrm{~A} \mathrm{MeV}$ bombarding energy are also included in Figure 2. The HADES data correspond to the $10 \%$ most central events [10]. The multiplicity values of HADES are not extrapolated over $4 \pi$, 
and by comparing them to adjacent FOPI multiplicity values (1200 A MeV) [5], we find that they are very different (for example, the FOPI proton multiplicity is 99.3 as compared to 77.6 for the HADES proton multiplicity). The figure shows, however, that the abundance ratio values are very close: HADES and FOPI results are compatible. We, therefore, see that the use of multiplicity ratios cancels out the detection inefficiencies.

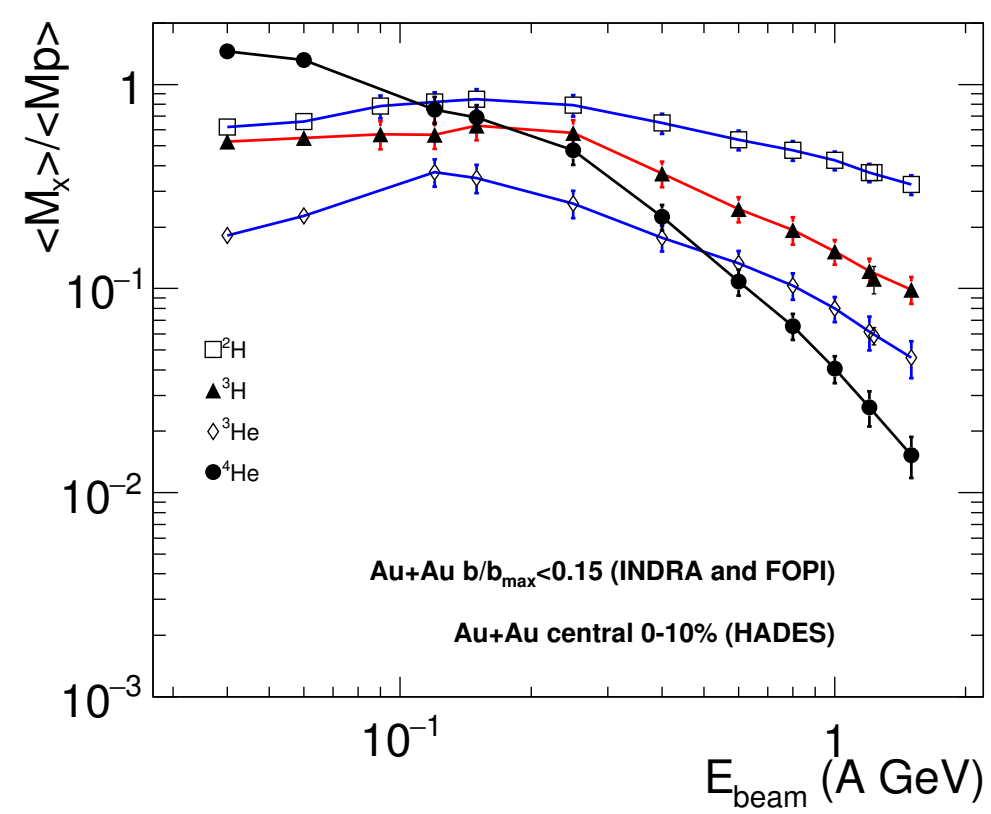

Figure 2. Light cluster abundance ratios as a function of bombarding energy for $\mathrm{Au}+\mathrm{Au}$. Lines to guide the eye. Line colours are to differentiate them.

Apart from the fact that the absolute values of cluster abundance ratios are not the same for $\mathrm{Au}+\mathrm{Au}$ and $\mathrm{Ni}+\mathrm{Ni} / \mathrm{Ca}+\mathrm{Ca}$ systems because of different isospin values, we can still observe some common trends between the two Figures. As the bombarding energy is increasing, we note a dramatic decrease in the ${ }^{4} \mathrm{He}$ abundance ratio. Simultaneously, the other cluster abundance ratios are increasing to a maximum value, which is reached at about $150 \mathrm{~A} \mathrm{MeV}$; this is particularly true for ${ }^{2} \mathrm{H}$. Above $200 \mathrm{~A} \mathrm{MeV}$, all cluster abundance ratios are decreasing with the increasing bombarding energy. The ${ }^{4} \mathrm{He}$ abundance ratio decreases even more rapidly compared to the others.

Different mechanisms of cluster production are at work in central heavy-ion collisions. In particular, at moderate bombarding energy, part of this production is due to secondary de-excitation from fragments produced with internal excitation energy. Nevertheless, the common trends observed in Figures 1 and 2 are significant.

\subsection{Cluster Production: Selected Multiplicity Ratios}

The ${ }^{3} \mathrm{H}$ and ${ }^{3} \mathrm{He}$ productions are strongly isospin-dependent [6]; this is also visible comparing Figures 1 and 2. We have plotted in Figure 3 the ratio of mean multiplicities of the two species for $\mathrm{Au}+\mathrm{Au}$ and $\mathrm{Ni}+\mathrm{Ni} / \mathrm{Ca}+\mathrm{Ca}$ systems. It is seen that the use of the ratio of mean multiplicities of ${ }^{3} \mathrm{H}$ and ${ }^{3} \mathrm{He}$ does not cancel the isospin dependence. The ratio is bigger for the $\mathrm{Au}+\mathrm{Au}$ system, whose isospin value is the greatest (see Table 1). For an ideal gas scenario, this ratio is related to the free-neutron-to-free-proton ratio [11]. This would imply a simultaneous emission of all species, thus ignoring the possible existence of a temporality in the lcp emission process [12-14]. For example, it has been experimentally shown that the characteristics of ${ }^{3} \mathrm{He}$ production reflect, on average, a rapid emission [6] at Fermi energies. It follows that this does not justify using this ratio to measure the ratio of free neutrons to free protons, which, at best, reflects it over the full energy range studied here. Therefore, this ratio will not be used here to measure a characteristic of a hypothetic 
lcp emission source but rather to give us a reference to compare the differences between other observables that will be studied next.

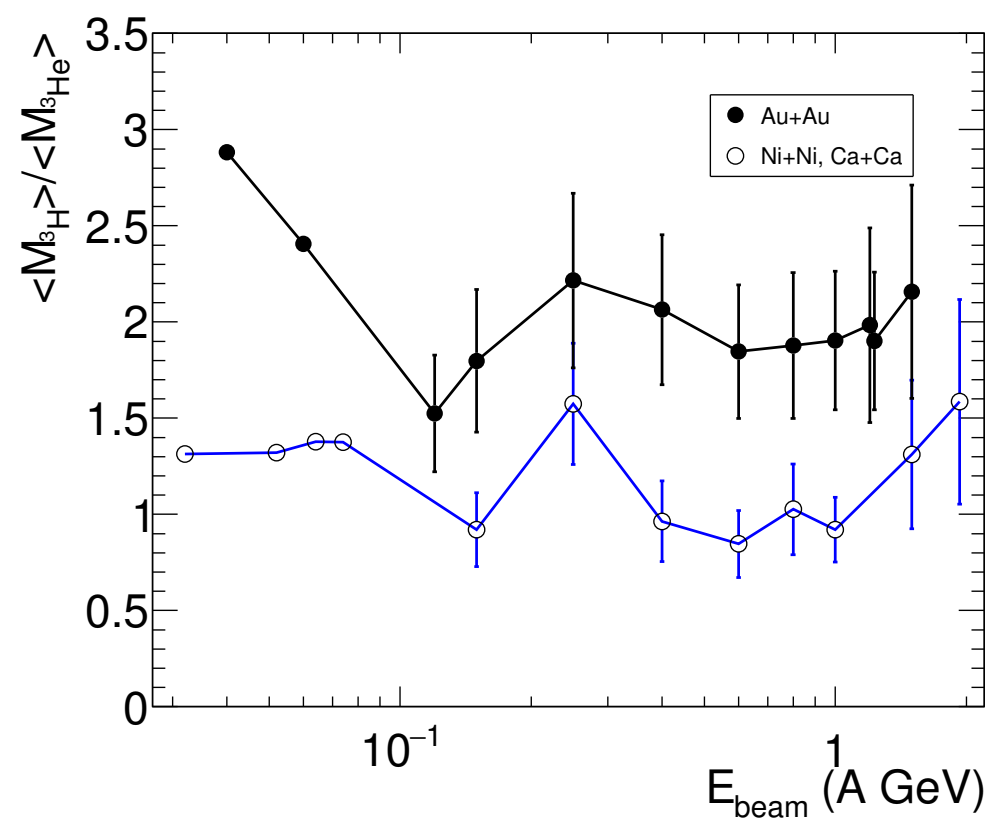

Figure 3. The ratio of ${ }^{3} \mathrm{H}$ to ${ }^{3} \mathrm{He}$ mean multiplicities as a function of bombarding energy for $\mathrm{Au}+\mathrm{Au}$ and $\mathrm{Ni}+\mathrm{Ni} / \mathrm{Ca}+\mathrm{Ca}$ systems. Lines to guide the eye. The black line corresponds to $\mathrm{Au}+\mathrm{Au}$, the blue line to $\mathrm{Ni}+\mathrm{Ni} / \mathrm{Ca}+\mathrm{Ca}$.

The ${ }^{4} \mathrm{He}$ to ${ }^{2} \mathrm{H}$ mean multiplicity ratio is now examined. This ratio is presented in Figure 4. For all systems, the ratio is decreasing with the increasing bombarding energy. This reflects the dramatic decrease in the ${ }^{4} \mathrm{He}$ abundance ratio. The ratio ${ }^{4} \mathrm{He} /{ }^{2} \mathrm{H}$ is almost system-independent from a few tens $\mathrm{A} \mathrm{MeV}$ up to about $150 \mathrm{~A} \mathrm{MeV}$, the value for which the ${ }^{2} \mathrm{H}$ abundance ratio reaches its maximum (Figures 1 and 2). Then increasing the bombarding energy, the two curves diverge to reach a relative difference close to the one observed for the ${ }^{3} \mathrm{H}$ over ${ }^{3} \mathrm{He}$ ratio (Figure 3). For low bombarding energies, ${ }^{4} \mathrm{He}$ can be considered as two ${ }^{2} \mathrm{H}$, and therefore, the ratio is almost independent of the system. At higher energies, this independence fades, and the heavier particle is less likely to be produced when the system is lighter. This indicates a change of light cluster mean production around a few hundred $\mathrm{A} \mathrm{MeV}$.

A combined ratio using ${ }^{2} \mathrm{H},{ }^{3} \mathrm{H},{ }^{3} \mathrm{He}$ and ${ }^{4} \mathrm{He}$ mean multiplicities will now be examined. It is presented in Figure 5 as a function of bombarding energy. The ratio with ${ }^{4} \mathrm{He}$ and ${ }^{2} \mathrm{H}$ mean multiplicities in the numerator and ${ }^{3} \mathrm{He}$ and ${ }^{3} \mathrm{H}$ mean multiplicities in the denominator is directly connected to the temperature of an ideal gas [11]. It should be isospin and mass-independent in this scenario. From Figure 5, according to bombarding energy, it can be seen: (i) From a few tens of $\mathrm{A} \mathrm{MeV}$, the ratio depends on the system characteristics; (ii) There is a change of slope around $150 \mathrm{~A} \mathrm{MeV;} \mathrm{(iii)} \mathrm{From} 400 \mathrm{~A} \mathrm{MeV}$ and above, the ratio is system independent. This independence for high bombarding energy is remarkable since, from Figures 3 and 4, it has been noted a system dependency for ${ }^{3} \mathrm{H} /{ }^{3} \mathrm{He}$ and ${ }^{4} \mathrm{He} /{ }^{2} \mathrm{H}$. All the mean multiplicity differences counterbalance from $400 \mathrm{~A} \mathrm{MeV}$. This could be an indication that, on average, all detected particles are emitted simultaneously from $400 \mathrm{~A} \mathrm{MeV}$ onwards, knowing that, for lower energies, the average values contain the imprint of different processes. This modification starts to be observed around $150 \mathrm{~A}$ $\mathrm{MeV}$ by a change in the slope, the bombarding energy value for which the production of ${ }^{2} \mathrm{H}$ reaches a maximum. 


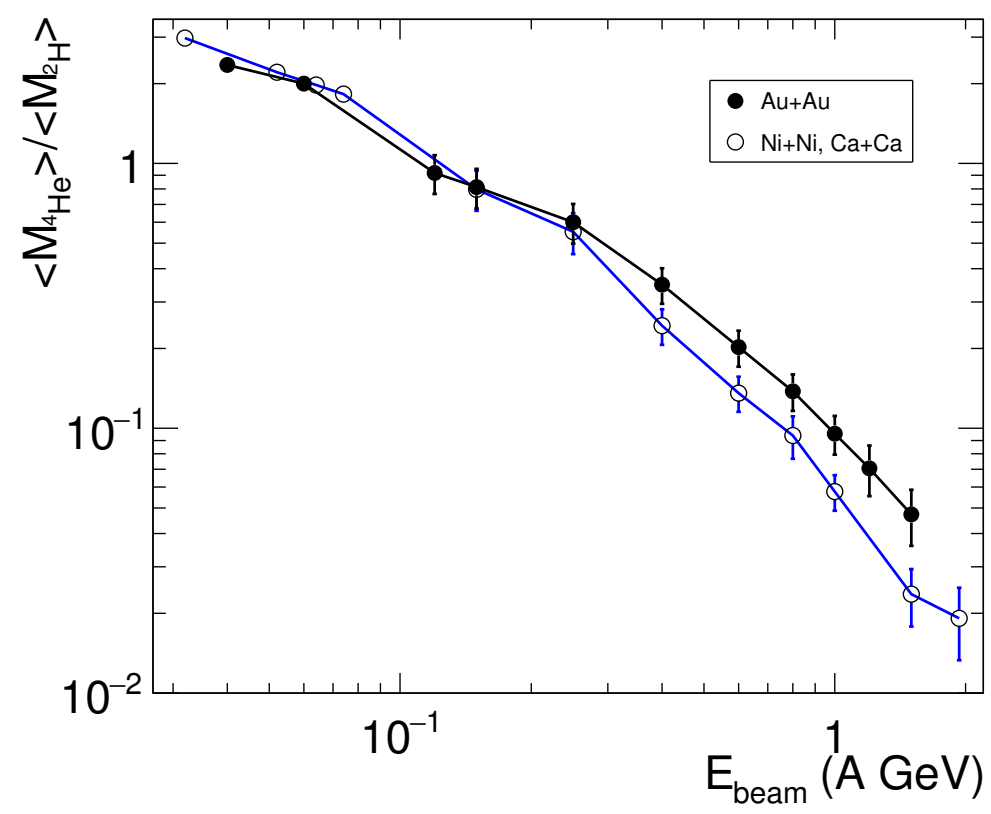

Figure 4. The ratio of ${ }^{4} \mathrm{He}$ to ${ }^{2} \mathrm{H}$ mean multiplicities as a function of bombarding energy for $\mathrm{Au}+\mathrm{Au}$ and $\mathrm{Ni}+\mathrm{Ni} / \mathrm{Ca}+\mathrm{Ca}$ systems. Lines to guide the eye. The black line corresponds to $\mathrm{Au}+\mathrm{Au}$, the blue line to $\mathrm{Ni}+\mathrm{Ni} / \mathrm{Ca}+\mathrm{Ca}$.

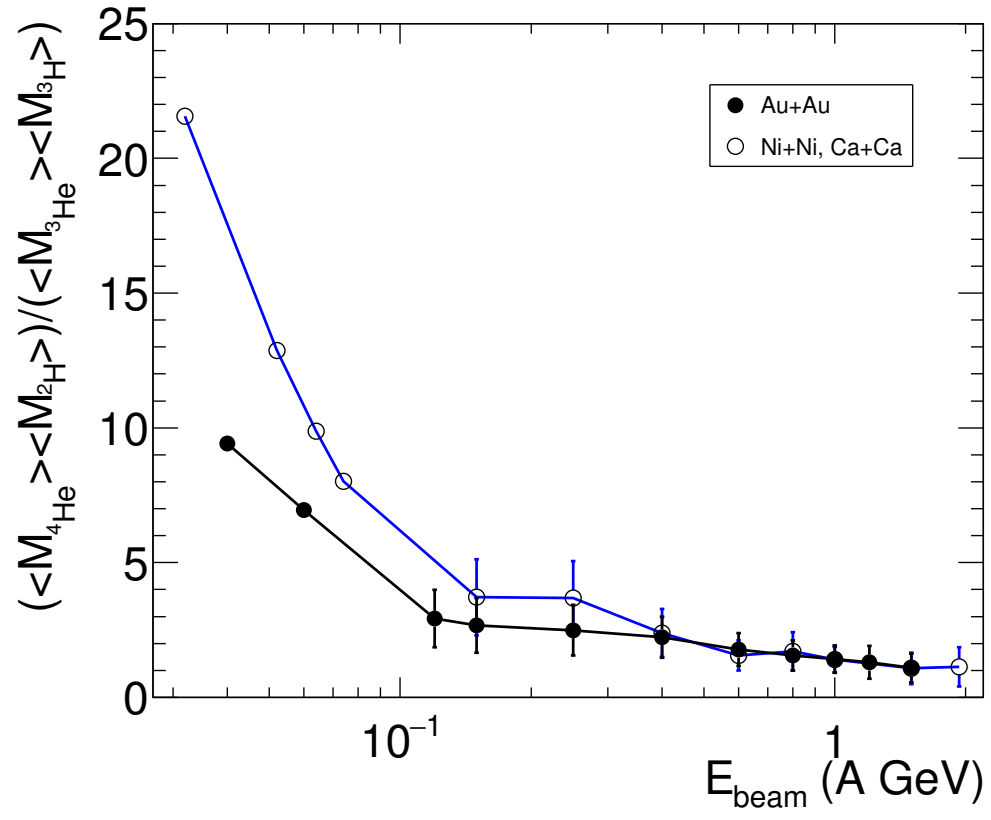

Figure 5. The combined ratio using ${ }^{2} \mathrm{H},{ }^{3} \mathrm{H},{ }^{3} \mathrm{He}$ and ${ }^{4} \mathrm{He}$ mean multiplicities as a function of bombarding energy for $\mathrm{Au}+\mathrm{Au}$ and $\mathrm{Ni}+\mathrm{Ni} / \mathrm{Ca}+\mathrm{Ca}$ systems. Lines to guide the eye. The black line corresponds to $\mathrm{Au}+\mathrm{Au}$, the blue line to $\mathrm{Ni}+\mathrm{Ni} / \mathrm{Ca}+\mathrm{Ca}$.

\section{Discussion}

The aim of the present article is to provide experimental data on cluster production in central collisions to which the model calculations can be directly compared. For this purpose, the FOPI data of [5] were extended to lower bombarding energies using data obtained with INDRA multidetector both at GANIL (Caen) and GSI (Darmstadt). The results are presented in the form of ratios in order to be less dependent on the experimental set-up and different bombarding energies. The ratios are light cluster abundance ratios, 
i.e., the mean cluster multiplicity over the mean proton multiplicity. Furthermore, the following ratios are presented: ${ }^{3} \mathrm{H} /{ }^{3} \mathrm{He},{ }^{4} \mathrm{He} /{ }^{2} \mathrm{H}$ and ${ }^{4} \mathrm{He}^{2} \mathrm{H} /{ }^{3} \mathrm{He}{ }^{3} \mathrm{H}\left({ }^{A} \mathrm{X}\right.$ represents the mean value of the cluster multiplicity). The extension towards low bombarding energies allows highlighting common features concerning cluster production for $\mathrm{Au}+\mathrm{Au}$ and combined $\mathrm{Ni}+\mathrm{Ni} / \mathrm{Ca}+\mathrm{Ca}$ systems, which are different systems in regards to total mass and total neutron-to-proton ratio. When increasing the bombarding energy for all systems:

1. There is a dramatic decrease in the ${ }^{4} \mathrm{He}$ abundance ratio;

2. There exists a clear maximum for the ${ }^{2} \mathrm{H}$ abundance ratio located at around $150 \mathrm{~A}$ $\mathrm{MeV}$;

3. There also exists a maximum around $150 \mathrm{~A} \mathrm{MeV} \mathrm{for}{ }^{3} \mathrm{H}$ and ${ }^{3} \mathrm{He}$ abundance ratios, but this maximum is less pronounced.

Looking at ${ }^{4} \mathrm{He} /{ }^{2} \mathrm{H}$ and ${ }^{4} \mathrm{He}^{2} \mathrm{H} /{ }^{3} \mathrm{He}^{3} \mathrm{H}$ ratios, it appears that there exists a change of the mechanism of cluster production for that maximum value of $150 \mathrm{~A} \mathrm{MeV}$. For low bombarding energies, several mechanisms compete, including direct production and secondary decay of excited fragments, whereas for high bombarding energies, it seems that, on average, the cluster production is more in line with a common temporality.

The presented results concern static observables (multiplicity), and they should be seen in conjunction with the results obtained on nuclear stopping in central events $[5,15,16]$ concerning dynamical observables. Cluster production modelling needs to take both aspects into account.

Author Contributions: Investigation, R.B., B.B., A.C., Q.F., J.D.F., E.G., T.G., D.G., M.H., M.L.C., N.L.N., I.L., O.L., M.P., P.P., G.V., E.V. and M.V.; writing—original draft preparation, R.B. All authors have read and agreed to the published version of the manuscript.

Funding: We acknowledge support from the Centre National de la Recherche Scientifique/Institut National de Physique Nucléaire et de Physique des Particules (France) and Région Normandie (France) under RIN/FIDNEOS.

Institutional Review Board Statement: Not applicable.

Informed Consent Statement: Not applicable.

Data Availability Statement: Not applicable.

Acknowledgments: This work is part of the INDRA collaboration program. We thank the GANIL and GSI staff for providing us the beams and for the technical support during the experiments.

Conflicts of Interest: The authors declare no conflict of interest. The funders had no role in the design of the study; in the collection, analyses, or interpretation of data; in the writing of the manuscript, or in the decision to publish the results.

\section{References}

1. Ono, A. Dynamics of clusters and fragments in heavy-ion collisions. Prog. Part. Nucl. Phys. 2019, 105, 139-179. [CrossRef]

2. Danielewicz, P.; Pan, Q. Blast of light fragments from central heavy-ion collisions. Phys. Rev. C 1992, 46, 2002-2011. [CrossRef] [PubMed]

3. Pais, H.; Bougault, R.; Gulminelli, F.; Providência, C.; Bonnet, E.; Borderie, B.; Chbihi, A.; Frankland, J.D.; Galichet, E.; Gruyer, D.; et al. Low Density In-Medium Effects on Light Clusters from Heavy-Ion Data. Phys. Rev. Lett. 2020, 125, 012701. [CrossRef] [PubMed]

4. Pouthas, J.O.E.L.; Borderie, B.; Dayras, R.; Plagnol, E.; Rivet, M.F.; Saint-Laurent, F.; Steckmeyer, J.C.; Auger, G.; Bacri, C.O.; Barbey, S.; et al. INDRA, a $4 \pi$ charged product detection array at GANIL. Nucl. Instrum. Methods Phys. Res. Sect. A 1995, 357, 418-442. [CrossRef]

5. Reisdorf, W.; Andronic, A.; Averbeck, R.; Benabderrahmane, M.L.; Hartmann, O.N.; Herrmann, N.; Hildenbrand, K.D.; Kang, T.I.; Kim, Y.J.; Kiš, M.; et al. Systematics of central heavy ion collisions in the $1 \mathrm{~A} \mathrm{GeV} \mathrm{regime.} \mathrm{Nucl.} \mathrm{Phys.} \mathrm{A} \mathrm{2010,} \mathrm{848,} \mathrm{366-427.}$ [CrossRef]

6. Bougault, R.; Bonnet, E.; Borderie, B.; Chbihi, A.; Dell'Aquila, D.; Fable, Q.; Francalanza, L.; Frankland, J.; Galichet, E.; Gruyer, D.; et al. Light charged clusters emitted in $32 \mathrm{MeV} /$ nucleon ${ }^{136,124} \mathrm{Xe}+{ }^{124,112} \mathrm{Sn}$ reactions: Chemical equilibrium and production of ${ }^{3} \mathrm{He}$ and ${ }^{6} \mathrm{He}$. Phys. Rev. C 2018, 97, 024612. [CrossRef] 
7. Cavata, C.; Demoulins, M.; Gosset, J.; Lemaire, M.-C.; L'Hôte, D.; Poitou, J.; Valette, O. Determination of the impact parameter in relativistic nucleus-nucleus collisions. Phys. Rev. C 1990, 42, 1760-1763. [CrossRef] [PubMed]

8. Gutbrod, H.H.; Poskanzer, A.M.; Ritter, H.G. Plastic Ball experiments. Rep. Prog. Phys. 1989, 52, 1267-1328. [CrossRef]

9. ECT* Workshop: Light Clusters in Nuclei and Nuclear Matter: Nuclear Structure and Decay, Heavy Ion Collisions, and Astrophysics. 2019. Available online: https://indico.ectstar.eu/event/52/contributions / (accessed on 15 July 2020).

10. Adamczewski-Musch, J.; HADES Collaboration; Arnold, O.; Behnke, C.; Belounnas, A.; Belyaev, A.; Berger-Chen, J.C.; Biernat, J.; Blanco, A.; Blume, C.; et al. Centrality determination of Au + Au collisions at 1.23A GeV with HADES. Eur. Phys. J. A 2018, 54, 85. [CrossRef]

11. Albergo, S.; Costa, S.; Costanzo, E.; Rubbino, A. Temperature and Free-Nucleon Densities of Nuclear Matter Exploding into Light Clusters in Heavy-Ion Collisions. Nuovo Cimento A 1985, 89, 1-28. [CrossRef]

12. Xi, H.F.; Kunde, G.J.; Bjarki, O.; Gelbke, C.K.; Lemmon, R.C.; Lynch, W.; Magestro, D.; Popescu, R.; Shomin, R.; Tsang, M.B.; et al. Dynamical emission and isotope thermometry. Phys. Rev. C 1998, 58, R2636-R2639. [CrossRef]

13. Verde, G.; Brown, D.A.; Danielewicz, P.; Gelbke, C.K.; Lynch, W.; Tsang, M.B. Imaging sources with fast and slow emission components. Phys. Rev. C 2002, 58, 054609. [CrossRef]

14. Chen, L.-W.; Ko, C.; Li, B.-A. Light cluster production in intermediate energy heavy-ion collisions induced by neutron-rich nuclei. Nucl. Phys. A 2003, 729, 809-834. [CrossRef]

15. Andronic, A.; Łukasik, J.; Reisdorf, W.; Trautmann, W. Systematics of stopping and flow in Au + Au collisions. Eur. Phys. J. A 2006, 30, 31-46. [CrossRef]

16. Henri, M.; Lopez, O.; Durand, D.; Borderie, B.; Bougault, R.; Chbihi, A.; Fable, Q.; Frankland, J.D.; Galichet, E.; Gruyer, D.; et al. In-medium effects in central heavy ion collisions at intermediate energies. Phys. Rev. C 2020, 101, 064622. [CrossRef] 